\title{
METABOLIC SYNDROME, POLYCYSTIC OVARY SYNDROME AND THE IMPORTANCE OF BEING EARNEST IN DEFINING THE MOST COMMON ENDOCRINE DISEASES. AND LET'S NOT FORGET ABOUT MEN - THEY ARE ALSO IMPORTANT!
}

\author{
M. Boyanov ${ }^{1}$ and M. Vankova ${ }^{2}$ \\ ${ }^{1}$ Clinic of Endocrinology and Metabolic Diseases, Department of Internal Diseases, \\ University Hospital "Alexandrovska", Sofia, Bulgaria \\ ${ }^{2}$ Endocrinology Unit, First Clinic of Internal Diseases, Tokuda Hospital, Sofia, Bulgaria
}

A $\mathrm{s}$ literature has proven the importance of being earnest, it is time that the medical society, and especially the endocrine societies worldwide, focus on how important it is to finally define our diseases, as this is an obligatory step in the diagnostic work-up, not to forget treatment, and foremost - prevention of any disease. Dedicated involvement in the essence of pathophysiology is a prerequisite for actually figuring out what is hidden beyond the poorly defined syndromes that we desperately look for in our routine clinical practice.

In the past several decades physicians have followed their intuition in the management of patients at risk of developing diabetes mellitus type 2. They would perceive the invisible interaction between insulin resistance, non-alcoholic fatty liver disease, obstructive sleep apnea (these have never been included in the definition of the metabolic syndrome), dyslipidemia, elevated fasting blood glucose and diabetes mellitus type 2. In fact, insulin resistance never got the opportunity to acquire a definition [1]. Defining insulin resistance would readily recall the need for measuring waist circumstance (a method known for its unreliability due to lack of standards regarding methodology of measurement and uncertainty in its reproducibility [2]). A simple but applicable definition would allow researchers to unify inclusion criteria used in clinical trials and make results more comparable. Diagnostic criteria for insulin resistance may differ from the cut-off where medical treatment is needed or the cut-off may be lower when other endocrine disorders are present. High risk individuals prone to developing prediabetes or diabetes should be identified in light of their co-morbidities and then accordingly treated. Since the last scientific statement for establishing the diagnosis of the metabolic syndrome came into force in 2009 [3], and was widely accepted thereafter, there was not a single organization to propose a more sensitive tool in identifying patients at risk. In 2014, the members of the American Association of Clinical Endocrinologists finally raised their voices to tell the world that it's just not that simple, proposing a quite complicated, but very comprehensive classification of obesity [4]. Just as a reminder, obesity was also recently defined as a disease, in June, 2013 [5]. One might think that our aim, as physicians, is to increase the number of diseases, and 
therefore, of people that would receive a diagnosis and, eventually, seek treatment. After all, if that was the aim, why to bother making a difference between obesity and the so-called "healthy obesity"? Actually, the aim is quite different. Scientists are seeking for the first pathophysiologic step in order to interrupt it, because they know what follows after and want to change the course of the disease. Just recently, Phillips L. S. et al. presented an intriguing idea that we could change the natural history of type 2 diabetes [6]. Possibly, we might change the course of any chronic disease, if we diagnose it early.

The common instinct of an experienced physician is from where science begins. Not all obese people would develop glucose intolerance in the future. Ask a practicing physician if they have seen a so-called "morbidly" obese individual with a perfectly normal fasting blood glucose and it would not be surprising that that phenomenon would be quite common, but just recently it received a name, the so-called "metabolically healthy obesity" [7]. Even though we call it "metabolically healthy", the case should not be closed, as the final judge for that individual would not be favourable at all, if left without appropriate treatment. To let this patient leave our office with no further instructions would make the phrase "think about the individual patient" sound hypocritical.

Patients with obesity should be evaluated for depression and arthritis before being accused of having a sedentary lifestyle [4], as should women with polycystic ovary syndrome (PCOS) be assessed for risk of deep venous thrombosis and mood disorders before receiving a prescription for any oral contraceptive. Many prescriptions are based on perception rather than evidence-based medicine [8].

PCOS is another example of a syndrome which has been poorly defined. The pathophysiologic concept of the syndrome is unclear, so is its treatment. Large numbers of women have been assigned the PCOS label. Many of them would benefit from treatment with metformin and conceive, but, unfortunately metformin is not allowed during pregnancy. Moreover, according to the Endocrine Society's most recent recommendation [9], metformin should be reserved for those who are contraindicated to receive oral contraceptives, i.e. as second-line therapy.

Metformin is probably the only medicine for the prevention of diabetes, yet not approved by the FDA for that specific indication [10]; it's a well-tolerated and effective drug in most cases of PCOS and is useful in reducing weight, with a mean of about $4 \mathrm{~kg}$, when dealing with "pure" obesity. Patients with obesity often have hepatic steatosis and dyslipidemia, and metformin is beneficial in both. In fact, obesity is another indication not included in the approved labelling of the drug. Gregory A. Nichols asked in December, 2014, should everyone take metformin [11]. A good answer to that would be - who should not? That would be a practical question to raise, and indeed, recommendations in the field are scarce.

Furthermore, preventing a syndrome and treating a disease with all related complications are two quite different tasks. Recommendations on how to manage diabetic patients receiving chronic haemodialysis are one unmet need. Guidance to how to manage uncontrolled diabetes in patients with recurring peripheral macrovascular occlusions and a number of other comorbidities might be also helpful for the practice.

Finally, we need to share some thoughts on the male gender. In fact, men are so neglected in endocrinology, that they are almost always diagnosed directly with diabetes or, if they are lucky, with all five criteria for the metabolic syndrome fulfilled. They are less frequently examined because they avoid hospitals to the very end. They are less frequently screened for thyroid diseases as they are less likely to ever get pregnant and thyroid cancer is less likely to incidentally be found during an ultrasound examination, as such examination is generally performed when you are able to palpate the thyroid gland. Osteoporosis would also be looked for in the female gender on the first place, but men could also be affected. 
Going back to basics is one thing one should do once in a while. A novel insight to prediseases may be compared to those musicians and artists of the 18th and 19th century, who could think of every little detail and still produce a composite whole not a puzzle. The precision of their performance, no matter what they were performing - painting The Starry Night, playing The Moonlight Sonata, sculpturing The Gates of Hell, is what science may need. They were earnest enough in their job. And are we?

\title{
REFERENCES:
}

1. Olatunbosun, S. T., Griffing G. T., et al. Insulin Resistance. Medscape Reference. Updated: March 8, 2013. Available at: http://emedicine.medscape.com/article/122501-overview, Accessed: Jan 30, 2015.

2. Agarwal, S. K., Misra A., Aggarwal P. et al. Waist Circumference Measurement by Site, Posture, Respiratory Phase, and Meal Time: Implications for Methodology. Obesity, 17, 2009, 1056-1061.

3. Alberti, K. G. M. M, Eckel, R. H., Grundy et al. Harmonizing the Metabolic Syndrome A Joint Interim Statement of the International Diabetes Federation Task Force on Epidemiology and Prevention; National Heart, Lung, and Blood Institute; American Heart Association; World Heart Federation; International Atherosclerosis Society; and International Association for the Study of Obesity. Circulation, 120, 2009, 1640-1645.

4. Garvey, W. T., Mechanick J. I., D. Einhorn. American Association of Clinical Endocrinologists and the American College of Endocrinology 2014 Advanced Framework For A New Diagnosis Of Obesity As A Chronic Disease. Available at: https://www.aace.com/files/2014-advanced-framework-for-a-new-diagnosis-of-obesity-as-a-chronic-disease.pdf (Accessed: Jan 29, 2015).

5. Frellick, M. AMA Declares Obesity a Disease. Medscape, 19, 2013.

6. Phillips, L. S., Ratner R. E., Buse J. B. et al. We Can Change the Natural History of Type 2 Diabetes. Diabetes Care, 37, 2014, 10, 2668-2676.

7. Pataky, Z., Bobbioni-Harsch E. et A. Golay. Open questions about metabolically normal obesity. International Journal of Obesity. 34, 2010, S18-S23.

8. Legro, R. S. Evaluation and Treatment of Polycystic Ovary Syndrome. Updated: September 19, 2009. Available at: http://www.endotext.org/chapter/evaluation-and-treatment-of-polycystic-ovary-syndrome (Accessed: Jan 29, 2015)

9. Legro, R. S., Arslanian S. A., Ehrmann D. A. et al. Diagnosis and Treatment of Polycystic Ovary Syndrome: An Endocrine Society Clinical Practice Guideline. J. Clin. Endocrinol. Metab., 98, 2013, 4565-4592. DOI: http:// dx.doi.org/10.1210/jc.2013-2350.

10. http://www.accessdata.fda.gov/drugsatfda_docs/label/2008/020357s031,021202s016lbl.pdf (Accessed Jan 30, 2015)

11. Nichols, G. A. Should Everyone Take Metformin? Medscape. Dec 04, 2014. Available at: http://www.medscape. com/viewarticle/835676 (Accessed: Jan 30, 2015).

\author{
Corresponding author: \\ Mira Vankova, MD \\ Endocrinology Unit \\ First Clinic of Internal Diseases \\ Tokuda Hospital Sofia \\ 51B Nikola Vaptsarov Blvd. \\ Sofia, Bulgaria \\ e-mail: miravankova@abv.bg
}

